\title{
Doing und Undoing Gender in Kinderkrippen: Eine Videostudie zu den Interaktionen von Kinderbetreuenden mit Kindern
}

\section{Franziska Vogt, Julia Nentwich und Wiebke Tennhoff}

Kinderkrippen sind für viele Kinder die erste Bildungsinstitution und für die Gleichstellung der Geschlechter bedeutsam. Im Beitrag wird auf der Basis einer ethnographischen Videostudie in vier Deutschschweizer Kinderkrippen untersucht, wie Gender in der pädagogischen Alltagspraxis der Kinderbetreuenden relevant wird. Für die Kodierung der Videodaten werden Interaktionsverläufe in Bezug auf doing und undoing gender, Dramatisierung und Dethematisierung analysiert. Die Ergebnisse zeigen, dass die Kinderbetreuenden das von Kindern gezeigte Verhalten, sei es doing oder undoing gender, verstärken, jedoch selten intervenieren um Gleichstellung herzustellen. Zur Förderung der Gleichstellung in der Kita sind die Organisationskultur und die pädagogische Qualität entscheidend.

\section{Einle itung}

Immer mehr Kinder machen ihre ersten Erfahrungen mit Bildungsinstitutionen nicht erst im Alter von vier oder fünf Jahren im Kindergarten, sondern bereits früher: Rund 60\% der unter Fünfjährigen besuchen in der Schweiz eine Kita (OECD, 2012, S. 345), die Zahl der Kinderbetreuungsplätze hat stark zugenommen (Kommission für Wissenschaft, Bildung und Kultur des Nationalrates, 12.08.2014), der Bedarf ist jedoch noch nicht gedeckt (SKBF, 2014). Die Thematik der Kinderbetreuung ist sehr relevant für die Gleichstellung der Geschlechter (NFP60 Synthesebericht, 2014). So heben Stern et al. (2013) die Bedeutung familienergänzender Kinderbetreuung sowohl für die Erwerbstätigkeit von Müttern als auch für die Stärkung aktiver Väter in der Kinderbetreuung hervor. Neben der Frage des Angebotes stehen in der Schweiz auch vermehrt Fragen bezüglich des Bildungs- und Betreuungsauftrags wie auch zur pädagogischen Qualität im Zentrum (Vogt, im Druck). Dies spiegelt sich besonders in der Ausarbeitung eines Orientierungsrahmens für frühkindliche Bildung, Betreuung und Erziehung in der Schweiz (Wustmann Seiler \& Simoni, 2012) und eines Qualitätshandbuches für Kitas, verbunden mit einer Zertifi- 
zierung (Quali-Kita, 2014). Gender und geschlechterreflektierende Pädagogik werden in diesen Grundlagedokumenten für Kitas jedoch nicht thematisiert. Wir identifizieren hier für die Schweiz Forschungsbedarf: Während international einzelne Studien zu Gender in der Kita zu verzeichnen sind, besteht für die Schweiz bisher dazu keine empirische Forschung. Darum untersucht das im Rahmen des Nationalen Forschungsprogramms NFP60 durchgeführte Forschungsprojekt Puppenstuben, Bauecken und Waldtage: (un)doing gender in Kinderkrippen (Nentwich \& Vogt, 2014) ${ }^{1}$ drei Bereiche: (i) die institutionellen Arrangements und die Konstruktion von Geschlecht in den pädagogischen Konzepten, Ausbildungsinhalten, Konzeptionen professionellen Handelns und im räumlichen Angebot (ii), die Konstruktion von Geschlecht in den Interaktionen und (iii) die Perspektive auf Männer als Kinderbetreuer. In diesem Beitrag liegt der Fokus auf den Interaktionen zwischen Kinderbetreuenden und Kindern: Wie wird Geschlecht in den Interaktionen zwischen Kinderbetreuenden und Kindern relevant gemacht? Weil das freie Spiel und die freie Tätigkeit für die Pädagogik der frühen Kindheit eine grosse Bedeutung haben (Wood, 2011), werden in diesem Beitrag Interaktionen im Kontext des Spiels und der freien Tätigkeit untersucht.

Der Artikel thematisiert zuerst Genderaspekte in der frühen Kindheit allgemein und in der pädagogischen Arbeit in Kitas oder Kinderkrippen (die Begriffe werden synonym verwendet). Weiter werden die verschiedenen Konzeptionen des doing und undoing gender diskutiert und das methodologische Vorgehen zur Videostudie wird dargelegt. Die Ergebnisse umfassen eine Beschreibung der Interaktionen zwischen Kinderbetreuenden und Kindern im Kontext des Spiels und der freien Tätigkeit sowie die Identifikation von unterschiedlichen Interaktionsverläufen. Der Artikel schliesst mit einer Diskussion der methodischen Herausforderungen der Videostudie sowie mit Schlussfolgerungen zu den Bemühungen um pädagogische Qualität und Gleichstellung in der Kita.

\section{Frühe Bildung und die Konstruktion von Geschlecht}

Die frühe Kindheit, besonders das Alter zwischen zwei und sechs Jahren, hat eine grosse Bedeutung für den Aufbau von Vorstellungen über Geschlecht. Kinder eignen sich in dieser Zeit Wissen über Geschlecht an. Zur Konstruktion dieses Geschlechterwissens interpretieren sie, wie die Erwachsenen auf sie und ihre Handlungen reagieren, und welche Normen sie dadurch setzen. Geschlechterwissen wird selten explizit, jedoch häufig implizit vermittelt (Wetterer, 2008). Beispielsweise kennen schon drei bis fünfjährige Kinder Geschlechterstereotype und nehmen an, dass nicht geschlechtertypische Spielzeugwahl von ihren Eltern kritisiert würde (Freeman, 2007). Es sind die alltäglichen Interkationen und 
Praktiken, die Kindern vermitteln, welche Bedeutung die Kategorie Geschlecht für eine Person in unserer Gesellschaft hat (Maihofer, 2002). So setzen Kinder sich besonders mit den geschlechterdifferenzierenden Praktiken im Kontext des institutionellen Rahmens von Zweigeschlechtlichkeit (Bilden, 1991) auseinander. Diese Sozialisationsprozesse sind vielschichtig (Henschel, 2014; Paseka, 2008), da auch die Konstruktion von Geschlecht aktiv, fluide und wechselnd geschieht. Die Erforschung der Konstruktion von Geschlecht muss deshalb dieser Dynamik und Kontextgebundenheit Rechnung tragen. Für die Schule zeigten die Arbeiten von Faulstich-Wieland, Weber und Willems (2004) sowie Breidenstein und Kelle (2003) beispielhaft auf, wie diese Prozesse der Geschlechterkonstruktion dynamisch in spezifische Kontexte eingebettet sind. Für die frühe Kindheit eignet sich der Blick auf das Spiel der Kinder besonders gut, um den dynamischen Prozess der Geschlechterkonstruktion zu fassen, so beispielsweise Browne (2004), Hyun und Choi (2004), Papadopoulou (2012) und Rendtorff (2011).

Die Art und Weise, wie Spielumgebungen gestaltet werden und wie das Spiel von den Erwachsenen unterstützt wird, ist deshalb für die Forschung zu Geschlecht und früher Kindheit von grossem Interesse. MacNaughton (2006) zeichnete mit Hilfe von ethnographischen Beobachtungen detailliert nach, wie Gender im Alltag des Kindergartens konstruiert wird. Chick, Heilman-Houser und Hunter (2002) zeigten, wie sehr in den Kitas durch die alltäglichen Interaktionen Geschlechterstereotype verstärkt werden. Ärlemalm-Hagsér (2010) fand die Verstärkung von Geschlechterstereotypen durch die Kinderbetreuenden in den ausgewählten Kitas in Schweden, dies obschon die Kita Gleichstellung explizit betonten. Kuger, Kluczniok, Sechtig und Smidt (2011) liessen Kinderbetreuende die Stärken und Schwächen von Kindern einschätzen und fanden, dass diese Einschätzung teilweise Geschlechterstereotypen entspricht. Auf Grund der Beobachtungsdaten der rund hundert Kinder zeigen sich jedoch viele Gemeinsamkeiten und kaum Unterschiede zwischen den Geschlechtern, in Bezug auf die von den Kindern gewählten Aktivitäten wie auch auf die Begleitung durch die Kinderbetreuenden (Kuger et al., 2011). Brandes, Andrä, Röseler und Schneider-Andrich (2013) untersuchten in einem experimentellen Design das pädagogische Handeln von weiblichen und männlichen Kinderbetreuenden. Den Kinderbetreuenden stand für eine zwanzigminütige Interaktion mit einem Kind der gleiche Materialkoffer zum Basteln zur Verfügung. Die Interaktion wurde videografiert und anschliessend mit Ratings eingeschätzt. Während sich nur wenige Unterschiede zwischen den Männern und Frauen zeigen, veränderte sich die Situation jedoch je nachdem, ob sie mit einem Mädchen oder einem Jungen interagierten. So sprachen Kinderbetreuende mit Jungen «eher sachlich-funktional» und mit Mädchen eher über «die Beziehung oder Persönliches» (Brandes et al., 2013, S. 40). Auch die verwendeten Materialien reflektierten Geschlechterstereotypen. Während diese Studie das Ziel verfolgte zu untersuchen, ob Männer, etwas anderes' in die Kita einbringen, zeigen die 
Befunde, dass die Herausforderungen für Kinderbetreuende eher darin liegt, nicht geschlechterstereotyp zu interagieren.

Um die Verstärkung von Geschlechterstereotypen zu durchbrechen, benötigen die pädagogischen Fachpersonen mehr Bewusstsein und Reflexion der eigenen Rolle (Giraldo \& Colyar, 2012), Gender-Trainings (Börding, 2009) oder Genderkompetenz (Grünewald-Huber \& von Gunten, 2009). FaulstichWieland (2001) wie auch Rabe-Kleberg (2003) fordern Gender-Mainstreaming für den Bereich der Kindertagesstätten. Einen wesentlichen Beitrag dazu leistete das europäische Projekt, Gender Loops', welches in einem Handbuch die praktische Umsetzung von geschlechterreflektierten Pädagogik in Kitas anregt (Krabel \& Cremers, 2008).

In dieser Studie steht die Erforschung der Prozesse der Konstruktion von Gender im Zentrum. Ziel ist es zu analysieren, wie Gender im Alltag der Kitas relevant gemacht wird, insbesondere in den Interaktionen zwischen Kinderbetreuenden und Kindern.

\section{Doing und undoing gender, Dramatisierung und Dethematisierung}

Ausgehend vom Verständnis von Geschlecht als einer fluiden kulturellen Ressource, die in Interaktionen konstruiert wird, und derer sich Kinder und Betreuende situationsabhängig bedienen, sollen nun die theoretischen Grundlagen für die Analyse der Interaktionen zwischen Kinderbetreuenden und Kindern diskutiert werden. Im Zentrum steht dabei das Konzept des doing gender.

Doing gender ist zunächst eine ethnographische Beobachtungskategorie, die es erlaubt zu fassen, wie sich «Subjekte handelnd eine Positionen aneignen» (Rendtorff, 2006, S. 137).

Doing gender verweist auf das Herstellen von Geschlecht in einer erworbenen Routine (West \& Zimmermann, 1987). Mit Deutsch (2007) kann doing gender als die Betonung der Geschlechterdifferenz gefasst werden, die in der Binarität der Zweigeschlechtlichkeit gefangen ist (Kelan, 2010). Die Betonung erfolgt durch bestimmte Tätigkeiten, Gesten und Symbole, die im Alltag als "Genderismen» zur Verfügung stehen (Goffman, 1994). Mit Rückgriff auf das kulturell-historische Wissen werden Geschlechterdifferenzen, die durch doing gender hergestellt werden, als natürlich wahrgenommen.

Dem doing gender, dem fortlaufenden Herstellen der Geschlechterunterscheidung, kann undoing gender entgegengesetzt werden. Hirschauer (2001) sieht undoing gender als eine Unterbrechung, eine Unterlassung, Geschlecht als Kategorie relevant zu machen. Butler (2004) hingegen versteht undoing gender aktiver, als Widerstand und als das Stören der Unterscheidungspraktiken, West und Zimmermann (2009) schreiben dem undoing gender die Veränderung der Normen zu. Deutsch (2007) schliesslich versteht undoing gender als das Heraus- 
fordern der Geschlechterhierarchie und die Betonung der Gleichheit. Diese Definition nach Deutsch (2007) - doing gender als Herstellen von Differenz, undoing gender als das Herstellen von Gleichheit - lässt sich für die empirische Forschung gut umsetzen, da sie es erlaubt, ein Raster für die Bewertung von Alltagspraktiken zu entwickeln. Zwar wird die Veränderbarkeit und Performativität von doing und undoing gender nicht im ganzen Facettenreichtum erfasst, die Definition ist jedoch für eine Sichtung dessen, was an doing und undoing gender in den alltäglichen Praktiken geschieht, empirisch äusserst hilfreich und fruchtbar. In dieser Lesart beziehen sich doing und undoing gender auf die Zugehörigkeit zu einem Geschlecht: Wie Hollander (2012) aufbauend auf West und Zimmermann (2009) mit dem Konzept der accountability ausführt, werden Personen in Situationen jeweils zur Rechenschaft gezogen und dahingehend überprüft, ob das Verhalten den Erwartungen an die sex category entspricht.

Allgemein birgt das Begriffspaar des doing und undoing gender das Potenzial, den interaktiven Charakter der Konstruktion von Geschlecht zu betonen. Nentwich und Kelan (2014) zeigen auf, wie dieser Wechsel zwischen doing und undoing schnell und fluide geschieht. Eine Handlung kann situativ als doing gender interpretiert werden, dann aber zum undoing gender werden. Gherardi (1995) zeigt mit der Analyse von ceremonial work und remedial work, wie in der Abfolge der Interaktion Differenz zelebriert oder geheilt wird. Diese Wechsel als Teil der alltäglichen Praktiken im Bereich der Erziehung haben FaulstichWieland et al. (2004) in ihrer Analyse zu Gender in der Schule detailliert aufgezeigt. Zudem bringen sie in Anlehnung an Goffman (1994) die Begriffe Dramatisierung und Entdramatisierung ein. Dramatisieren bedeutet nach Goffman das Betonen und Hervorheben der Geschlechterdifferenz, Entdramatisierung wird von Faulstich-Wieland et al. (2004) als Ruhenlassen von Geschlechterdifferenz verstanden. Hirschauer (2001) hingegen schlägt den Begriff Dethematisierung vor: Während doing gender die Binarität von Geschlecht in den Mittelpunkt stellt, wird bei undoing gender diese nicht beachtet, Geschlecht wird dethematisiert, wird nicht zum Thema gemacht.

Zusammenfassend schlagen wir für die Analyse der Interaktionen in der Kinderkrippe vor, doing und undoing gender dafür zu verwenden, inwieweit in einer momentanen Handlung die Geschlechterdifferenz entlang der aktuell geltenden Geschlechternormen bzw. der Geschlechterstereotypen betont wird (doing gender) oder inwieweit dieses historisch-kulturell zur Verfügung gestellte Wissen herausgefordert und umgedeutet wird, was längerfristig das Potenzial der Veränderung der Geschlechternormen oder der Aufgabe der Binarität der Geschlechterordnung mit sich bringt (undoing gender). Geschlechterstereotype sind die kulturell-symbolischen Ressourcen (Pickering, 2001), mit denen in Alltagshandlungen Geschlecht relevant gemacht werden kann. Auch wenn viele Personen heutzutage die in Geschlechterstereotypen transportierte binäre Unterschiedlichkeit der Geschlechter nicht zwingend als leitende Norm akzeptieren, sind diese Geschlechterstereotype nach wie vor stabile Wissensbestände. Um 
Tätigkeiten in der Kinderkrippe als dem Geschlechterstereotyp entsprechend oder widersprechend einzuteilen, kann auf die Stereotypenforschung (Pickering, 2001) sowie auf Analysen der Geschlechterkodierung bei Spielzeug (Rendtdorff, 2011) zurückgegriffen werden. Wenn ein Junge mit Autos spielt, würde dies als dem Geschlechterstereotyp entsprechend eingeschätzt, wird ein Mädchen dabei beobachtet, würde dies als nicht dem Stereotyp entsprechend wahrgenommen. Damit wird in dieser Zuordnung das biologische Geschlecht des interagierenden Kindes, das etwas tut, berücksichtigt, denn auf dieser Grundlage des biologischen Geschlechts (sex) wird entschieden, ob die Tätigkeit den Erwartungen (accountability) an das kulturelle Geschlecht (gender) entspricht und ob also die kulturell zugeschriebenen Geschlechterdifferenzen mit dieser Interaktion verstärkt oder aber umgedeutet werden. Basierend auf Goffman (1995) und Faulstich-Wieland et al. (2004) wird zudem einbezogen, ob Gender in dieser Situation dramatisiert wird, oder ob es, wie Hirschauer (2001) konzipiert, dethematisiert wird. Mit den Begriffen Dethematisierung und Dramatisierung lässt sich in der vorliegenden Studie verdeutlichen, wie Kinderbetreuende dem doing und undoing gender der Kinder begegnen.

\section{Methoden}

Um die Relevanz von Geschlecht in den Alltagspraktiken und insbesondere in den Interaktionen in den Kinderkrippen zu untersuchen, wurde ein ethnographisches, videogestütztes Vorgehen gewählt. Im Folgenden werden zunächst das Sample und danach die Videostudie in Bezug auf die Erhebung der Daten und der Analyse dargestellt.

\section{Stichprobe}

Am Projekt nahmen 20 Kinderkrippen in der Deutschschweiz teil, davon zehn Kinderkrippen mit mindestens einem ausgebildeten männlichen Kinderbetreuer. In allen Krippen wurden Interviews mit den Kita-Leitungen sowie Raumbegehungen durchgeführt. Aufgrund der Analyse dieser Daten wurden vier Kinderkrippen ausgewählt, welche mindestens einen ausgebildeten männlichen Kinderbetreuer im Team hatten und die sich durch unterschiedliche Teamzusammensetzungen, pädagogische Konzepte und Raumgestaltung auszeichneten. Mit diesem kontrastierenden, theoretischen Sampling sollte gewährleistet werden, dass für die ethnographische Beobachtung und Videobeobachtung möglichst unterschiedliche Fälle zur Verfügung stehen. Unter den vier Krippen für die ethnographische Forschung befand sich eine Krippe (Kita blau) mit einem fast ausgeglichenen Verhältnis von Männern und Frauen im Team, während bei den anderen Frauen die Mehrheit bilden. In einer der Krippen beobachteten wir die naturpädagogisch geführte Gruppe im Wald (Kita grün). Eine weitere (Kita rot) zeichnete sich durch eine an Montessori angelehnte Pädagogik sowie eine 
Raumgestaltung mit viel unstrukturiertem Material aus. Kita gelb ist in Bezug auf die Raumgestaltung und das Konzept prototypisch für viele der besuchten Kitas. In den Kitas wurden altersgemischte Gruppen beobachtet, Kinder zwischen zwei bis vier Jahren sind am stärksten vertreten.

\section{Design teilnehmende Beobachtung und Videostudie}

Jede Krippe wurde während vier ganzen Tagen besucht, in der Regel je zwei Tage hintereinander über zwei Wochen, bei einer Kita konnten lediglich drei Beobachtungstage stattfinden. Aus dem Forschungsteam waren jeweils zwei Forscherinnen anwesend; beide führten ethnographische Protokolle, die im Anschluss an die Beobachtung auf Grund der Notizen wie auch aus der Erinnerung niedergeschrieben und diskutiert wurden. So oft wie möglich wurde mit einer Videokamera das Geschehen aufgenommen. Dabei wurde abwechslungsweise ein Kinderbetreuer oder eine Kinderbetreuerin mit einem Funkmikrofon ausgerüstet, und die Kamera folgte deren Tätigkeit möglichst unauffällig aus der Distanz. Das Videomaterial im Umfang von 50 Stunden wurde mit Hilfe der Software Atlas.ti vollständig analysiert.

Für die hier vorgestellte Analyse wurde mit Hilfe des Eventsamplings (Ostrov $\&$ Hart, 2013) für die Fragestellung relevante und für die Kita typische Sequenzen ausgewählt. Diese Auswahl umfasst kurze, etwa einminütige Interaktionen bis zu etwa zehnminütigen Sequenzen. Für die nicht ausgewählten Sequenzen wurde ein Memo verfasst, welches zusammenfassend darstellt, was auf diesen Filmteilen zu sehen ist und warum diese nicht für eine nähere Analyse ausgewählt wurden. Jede ausgewählte Sequenz wurde in Bezug auf drei Aspekte kategorisiert: (i) Interaktion mit den Subkategorien Kinderbetreuer-Kind, Kinderbetreuerin Kind, Kind - Kind, Kinderbetreuende - Kinderbetreuende, und Forschende Feld, (ii) Raum und Material (Subkategorien Bauecke, draussen, grosses Rollenspiel und Verkleidung, Küchenecke, Malen, Ruhebereich und weitere) und (iii) Sozialform und Aktivität (Subkategorien angeleitete Gruppe, freies Spiel, Aufräumen, Essen und weitere). Für die Überprüfung der Intersubjektivität der Auswahl der Sequenzen und ihrer Kategorisierung wurden vier Aufnahmen von mindestens je einer halben Stunde von je einer weiteren Forscherin unabhängig kodiert. $\mathrm{Zu}$ jeder ausgewählten Sequenz wurde ein Memo mit einer ersten, interpretativen Beschreibung der Sequenz in Bezug auf Gender verfasst und dabei für die in der Situation Beteiligten doing und undoing gender beschrieben. Um zu entscheiden, ob Gender relevant gemacht wird (Dramatisierung) oder aber im Hintergrund verbleibt (Dethematisierung), wurde einbezogen, wie die Interaktion von den Kinderbetreuenden her weitergeführt wurde, wie weiter unten genauer dargestellt wird (Analyse Interaktionsverläufe). Zunächst soll der Datenkorpus der ausgewählten Sequenzen beschrieben werden. 


\section{Datenkorpus Videosequenzen}

In diesem Beitrag steht das pädagogische Handeln der Kinderbetreuerinnen und Kinderbetreuer im Fokus. Daher wurden aus dem Videomaterial jene Sequenzen ausgewählt, in denen eine Interaktion zwischen Erwachsenen und Kindern stattfindet (insgesamt 352 Sequenzen). Von diesen Sequenzen wurden wiederum nur diejenigen ausgewählt, die mit den Sub-Kategorien Sozialform freies Spiel / freie Tätigkeit oder den Sub-Kategorien Puppenecke, Rollenspiel und Bauecke kodiert waren, insgesamt rund 150 Sequenzen. Das Erkenntnisinteresse war die Analyse der Interaktionen der Kinderbetreuenden mit den Kindern, wie sie auf doing und undoing gender der Kinder reagieren. Daher wurden nur jene Sequenzen beibehalten, bei denen eine vom Kind ausgehende Handlung oder Äusserung bestand, auf die die Kinderbetreuenden in der Interaktion reagieren oder nicht reagieren konnten. Sequenzen, die durch die Kinderbetreuenden initiiert wurden, wurden für die hier vorgestellte Analyse weggelassen.

Nach dieser Bereinigung umfasst der Datenkorpus 119 Sequenzen, die sich in Bezug auf die Krippen, das Geschlecht der Kinderbetreuenden und das Geschlecht der Kinder, wie in Tabelle 1 aufgeführt, aufteilen.

Tabelle 1: Datenkorpus für die Analyse

\begin{tabular}{|l|c|c|c|c|c|}
\hline $\begin{array}{l}\text { Anzahl } \\
\text { Sequenzen }\end{array}$ & $\begin{array}{c}\text { Kinder- } \\
\text { betreuer mit } \\
\text { Jungen }\end{array}$ & $\begin{array}{c}\text { Kinder- } \\
\text { betreuer mit } \\
\text { Mädchen }\end{array}$ & $\begin{array}{c}\text { Kinder- } \\
\text { betreuerin mit } \\
\text { Jungen }\end{array}$ & $\begin{array}{c}\text { Kinder- } \\
\text { betreuerin mit } \\
\text { Mädchen }\end{array}$ & $\begin{array}{c}\text { Total } \\
\text { Sequenzen } \\
\text { pro Kita }\end{array}$ \\
\hline Kita gelb & 19 & 10 & 5 & 0 & 34 \\
\hline Kita rot & 7 & 4 & 4 & 9 & 24 \\
\hline Kita grün & 9 & 5 & 2 & 7 & 23 \\
\hline Kita blau & 7 & 14 & 10 & 7 & 38 \\
\hline Total & 42 & 33 & 21 & 23 & 119 \\
\hline
\end{tabular}

Es entfallen insgesamt 63 Sequenzen auf Interaktionen mit Jungen und 56 auf Interaktionen mit Mädchen. Es sind mehr Sequenzen mit Kinderbetreuern ( $\mathrm{n}=$ 75) als mit Kinderbetreuerinnen $(n=44)$ im Datenkorpus, weil in Kita gelb die Kinderbetreuerin zugleich Gruppenleiterin und stellvertretende Kitaleiterin ist und dadurch weniger häufig mit den Kindern tätig war.

\section{Analyse der Interaktionsverläufe}

Für die Analyse der Interaktionen in der Kinderkrippe schlagen wir im Rückgriff auf die oben dargestellten Konzeptionen vor, doing und undoing gender dafür zu verwenden, inwieweit in einer momentanen Handlung die Geschlechterdifferenz durch Geschlechterstereotypen betont wird (doing gender) oder inwieweit diese umgedeutet werden und Geschlechtergleichheit betont wird (undoing gender). In dieser Definition sind doing und undoing gender immer auch in Relation zum biologischen Geschlecht (sex) der handelnden Person wie auch des situativen Kontexts zu sehen. Um Tätigkeiten als dem Geschlechterstereotyp entsprechend oder widersprechend einzuteilen, wurde die Stereotypenforschung, Analysen der 
Geschlechterkodierung bei Spielzeug (Rendtdorff, 2011) sowie Aussagen aus den Interviews des Forschungsprojektes mit Krippenleitungen, Kinderbetreuerinnen und -betreuern über geschlechtertypische Erwartungen an Buben und Mädchen beigezogen.

Für den Interaktionsverlauf wird ebenfalls untersucht, wie etwas getan wird. Hier wird zwischen Dramatisierung und Dethematisierung unterschieden. Wird zum Beispiel eine geschlechterdifferenzierende Konnotierung der Situation explizit von den Kinderbetreuenden kommentiert, interpretieren wir dies als Dramatisierung, verläuft sie unbemerkt im Hintergrund, ordnen wir sie als Dethematisierung ein. Ebenso kann die Umdeutung und Herausforderung der Geschlechterstereotypen in den Vordergrund gestellt werden oder nicht zum Thema gemacht werden.

Wenn die Kinderbetreuenden mit Dethematisierung reagieren und Geschlecht nicht relevant gemacht wird, ist damit der Verlauf dieser Interaktion abgeschlossen. Wenn die Kinderbetreuenden mit Dramatisierung auf die Passung oder die fehlende Passung zwischen der Handlung und den Geschlechterstereotpyen, bezogen auf das biologische Geschlecht des Kindes, reagieren, haben die Kinderbetreuenden zwei Möglichkeiten: Sie können mit doing gender die Geschlechterstereotypen betonen und einfordern, oder sie können mit undoing gender eine Handlung bestärken oder einfordern, die nicht den Geschlechterstereotpyen entspricht. Diese Analyseschritte führen zur Unterscheidung von sechs Interaktionsverläufen (Abbildung 1).

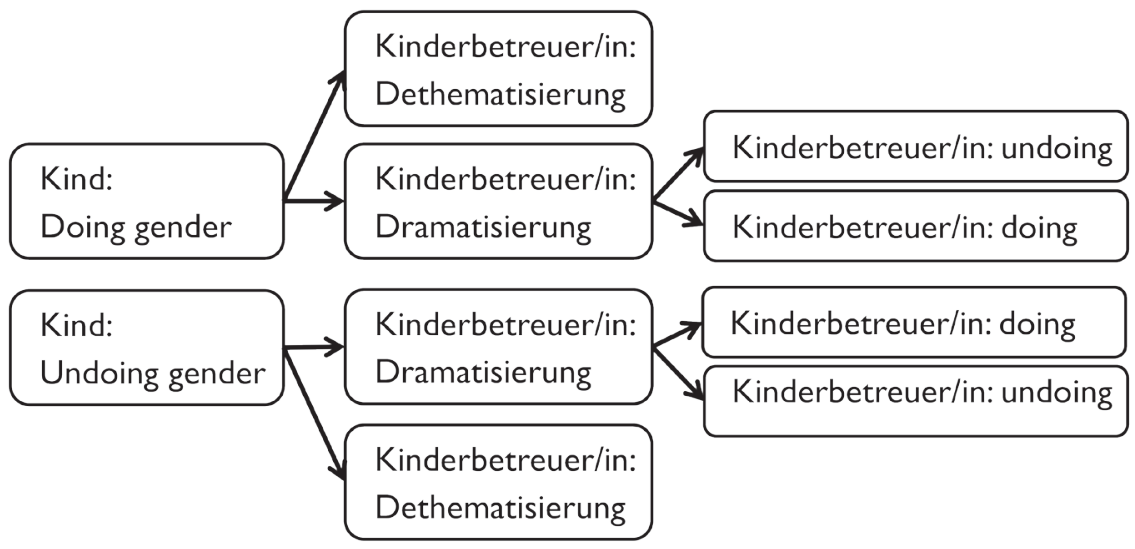

Abbildung 1: Interaktionsverläufe für die Interaktionen zwischen Kind und Kinderbetreuer/in in Bezug auf (un)doing gender und Dramatisierung / Dethematisierung. 
Für die Analyse des Datenkorpus von 119 Sequenzen aus dem freien Spiel und den freien Aktivitäten, bei denen eine Handlung vom Kind ausgeht und das Kind in Interaktion mit einer Kinderbetreuerin oder einem Kinderbetreuer steht, wurden Kategorien für diese sechs Interaktionsverläufe vergeben.

\section{Ergebnisse}

Zur Darstellung der Ergebnisse wird zunächst beschrieben, wie sich die theoretisch entwickelten sechs Interaktionsverläufe im Material zeigen. Dafür wird für jeden Interaktionsverlauf ein illustratives Beispiel dargestellt.

Kind doing gender, Kinderbetreuende Dethematisierung Dass ein Kind doing gender zeigt und die Kinderbetreuenden dies nicht beachten und dies so dethematisiert wird, tritt in sechs der Sequenzen dieses Datenkorpus auf. Häufig folgen hier die Kinderbetreuenden dem Spiel der Kinder oder nehmen hieran teil, ohne jedoch die hierin potentiell liegende geschlechtliche Konnotation aufzugreifen. Als Beispiel hier die Interaktion zwischen einem Kinderbetreuer und einem Jungen während der Freispielphase im Wald:

Der Junge schnitzt mit einem Taschenmesser am Stecken. Der Kinderbetreuer erklärt ihm in Worten, wie er das Messer halten soll, wie er schnitzen kann, und welche Regeln für das Schnitzen gelten. (Kita grün)

Mit dem Taschenmesser zu schnitzen entspricht den Geschlechterstereotypen für Jungen. Der Kinderbetreuer geht jedoch auf dieses doing gender nicht ein, sondern erklärt die Tätigkeit an sich, damit wird es dethematisiert.

\section{Kind doing gender, Kinderbetreuende Dramatisierung mit doing gender}

Auf diese Kategorie entfallen 34 Sequenzen. Als Beispiel ein Austausch eines Kinderbetreuers mit Jungen über Werkzeuge:

Ein Bub hält ein Werkzeug in der Hand, der Kinderbetreuer erklärt, was es ist und wofür es gebraucht wird. Die Buben nehmen Werkzeuge aus der Kiste und spielen damit. Der Kinderbetreuer sagt zu einem Buben: Da kommt der Baumann! (Kita grün)

Die Dramatisierung und doing gender wird hier mit dem Ausdruck, ‘Da kommt der Baumann, eine Anspielung auf die Kinderserie Bob der Baumeister, sichtbar. Das Beispiel zeigt exemplarisch, wie Gender in der Spielbegleitung häufig durch Zuschreibungen und dem Ausdruck von Erwartungen, was ein Kind ist oder sein könnte, relevant gemacht wird. In diesem Fall wird die dem Geschlechterstereotyp entsprechende Aktivität des Jungen, das Bauen, verstärkt, in dem er dem Jungen die Rolle des Baumanns zuschreibt. 
In einem weiteren Beispiel redet die Kinderbetreuerin mit einem Mädchen sehr lange über Mode, und ein Junge, der dazu kommt, wird nicht beachtet und nicht in die Interaktion integriert.

Die Kinderbetreuerin sitzt auf einer Bank und hat ein Mädchen auf dem Schoss. Sie trägt ein pinkes T-Shirt, darauf sind Schuhe abgebildet und es gibt einige Applikationen, wie Tüll und Pailletten, dazu einen Schriftzug Fashion makes me pretty. Das Mädchen nestelt am T-Shirt der Kinderbetreuerin, sie sagt: da sind Schuhe. Die Kinderbetreuerin präzisiert: Es sind Ballerinas. Das Mädchen zählt die Pailletten auf dem T-Shirt. Dann fragt sie, was auf dem T-Shirt steht, die Kinderbetreuerin übersetzt: Mode macht mich schön. (Kita blau)

Das Beispiel zeigt, wie der sogenannte, gender display' (Goffman, 1994) zur Aktivierung der Kategorie Geschlecht beiträgt und damit doing gender initiieren kann. Gender wird hier dramatisiert: Der Schriftzug wie auch die Kleidung der Betreuerin vermittelt eine stereotypisierende Botschaft, das Mädchen initiiert ein sehr langes Gespräch über das T-Shirt, auf das die Betreuerin fokussiert bleibt. Das Mädchen sitzt insgesamt während 25 Minuten auf dem Schoss der Betreuerin, andere im Raum anwesende Kinder werden nicht beachtet und nicht einbezogen. Die geschlechterstereotypen Inhalte werden durch die genaue Bezeichnung der Schuhe und durch die Übersetzung von fashion makes me pretty, dramatisiert. Die Aussage ,Mode macht mich schön', wird von der Betreuerin nicht diskutiert oder in Frage gestellt.

\section{Kind doing gender, Kinderbetreuende Dramatisierung mit undoing gender}

In dieser Kategorie befinden sich 14 Sequenzen, in denen dem mit stereotypen Erwartungen konforme Verhalten der Kinder mit einem dramatisierenden undoing gender der Betreuenden begegnet wird. Als Beispiel dient die Begleitung des Kinderbetreuers bei einer Aktivität auf einer Leiter:

Ein Mädchen und ein Junge klettern auf einer eigens errichteten Bogenleiter im Spielzimmer. Das Mädchen steigt vorsichtig auf der Leiter nach vorne, um dann hinunterzuklettern. Der Junge ist schon hinuntergeklettert und sagt zum Mädchen: «Hast du Angst?» Der Kinderbetreuer sagt zum Jungen: «Nein, sie will es anders.» (Kita rot)

Hier wird eine geschlechtsstereotype Deutung eines Kindes vom Kinderbetreuer thematisiert und umgedeutet und damit dramatisiert. Er greift die Interpretation des Jungen auf, indem er seiner geschlechterstereotypen Interpretation wiederspricht und eine alternative Interpretation des vorsichtigen Verhaltens des Mädchens anbietet. Während dem Geschlechterstereotyp gemäss «ängstlich sein» weiblich konnotiert wird, kontert der Kinderbetreuer hier diese Interpretation, was wir als undoing gender interpretieren. 
In dieser Kategorie finden sich einige Sequenzen, in denen Jungen aufgefordert werden, nicht wild und laut zu spielen, sondern ruhig zu sein. Die Reaktion auf dieses wilde Spiel mit der Betonung von Ordnungen, seien es Zeitordnungen oder Raumordnungen, kann als eine Betonung einer «hausfraulichen Weiblichkeit» (Rabe-Kleberg, 2003; Nentwich, Vogt \& Tennhoff, in Druck) interpretiert werden.

Nachdem nun drei Kategorien beschrieben wurden, die von einem doing gender des Kindes ausgehen, folgen nun die drei Interaktionsverläufe, bei denen ein undoing gender des Kindes am Anfang steht.

\section{Kind undoing gender, Kinderbetreuende Dramatisierung mit doing gender}

In dieser Kategorie befinden sich 14 Sequenzen. Als Beispiel dient eine Sequenz, bei der mehrere Jungen in der Puppenecke spielen. In der Puppenecke hat es unter anderem Stöckelschuhe als Requisiten.

Ein Junge sitzt neben dem Kinderbetreuer am Boden und zieht Stöckelschuhe an. Der Kinderbetreuer sagt zu ihm: "sieht lustig aus. Bist du s'Fräulein?» Junge: «Nein!» Kinderbetreuer: «Mit den Stöckelschuhen... Bist du das Mami?» Junge: «Tim ist das Mami.» (Kita gelb)

Die Handlung des Jungen, Stöckelschuhe anzuziehen, wird als undoing gender eingeschätzt. Der Kinderbetreuer weist in seiner Aussage jedoch nun auf die Diskrepanz zwischen Handlung und geschlechtsstereotypen Erwartungen hin und bietet dem Jungen weibliche Rollen, Fräulein und Mami, an. Im vorgestellten Interpretationsrahmen kann die Reaktion des Kinderbetreuers als Dramatisierung gelesen werden; der Umstand, dass hier die Nicht-Passung von symbolischem Geschlecht der Handlung und Geschlecht des Kindes mit einem weiblichen Rollenangebot (Fräulein, Mami) versehen wird, qualifiziert sie als doing gender. Der Eindruck der Dramatisierung wird dadurch verstärkt, dass der Ausdruck Fräulein eine kaum mehr gebräuchliche Bezeichnung für unverheiratete Frauen ist.

\section{Kind undoing gender, Kinderbetreuende Dramatisierung mit undoing gender}

In dieser Kategorie gibt es 40 Sequenzen, in denen die nicht dem Stereotyp entsprechenden Aktivitäten der Kinder durch die Reaktion der Kinderbetreuenden verstärkt werden. Im folgenden Beispiel unterstützt die Kinderbetreuerin das nichtstereotype Rollenspiel eines Mädchens:

Das Mädchen kommt zur Kinderbetreuerin und sagt, dass der Bauhelm kaputt sei. Die Kinderbetreuerin geht mit ihr zur Puppenecke zurück und sagt, es hätte noch einen andern. Sie finden einen Polizeihut, den das Mädchen haben möchte. Der erste Polizeihut ist zu gross, sie nimmt den zweiten und gibt den anderen der Kinderbetreuerin, die ihn auch aufsetzt. 
Die Kinderbetreuerin hilft dem Mädchen beim Anziehen der Jacke und sagt: «jetzt bist du eine Polizistin». (Kita rot)

Die Kinderbetreuerin unterstützt das Vorhaben des Mädchens, eine nicht geschlechterstereotype Rolle zu spielen und bietet ihr mit der Zuschreibung «Polizistin» auch eine Rolle zur Identifikation an.

Kind undoing gender, Kinderbetreuende Dethematisierung Bei diesen Sequenzen reagiert die Kinderbetreuerin oder der Kinderbetreuer nicht auf das vom Kind gezeigte untypische Verhalten. Insgesamt sind elf Sequenzen in dieser Kategorie. Als Beispiel dient eine Sequenz auf einer im Freien zum Spielen eingerichteten Baustelle:

Auf der Baustelle sitzt ein kleines Mädchen neben der Kinderbetreuerin und sägt, die Kinderbetreuerin beachtet es nicht, sie redet mit den Jungen, die auch sägen. (Kita grün)

Das Mädchen beschäftigt sich mit einem Werkzeug, das der geschlechtsstereotypen Erwartung widerspricht und darum als undoing gender interpretiert werden kann. Dies wird jedoch weder kommentiert noch anderweitig besonders beachtet.

Zusammenfassend zeigt sich in den Interaktionsverläufen, dass Gender in der Kita in sehr unterschiedlicher Art und Weise relevant gemacht wird. Nach dieser detaillierteren Beschreibung wird nun im Überblick gezeigt, welche Muster es in der Verteilung der Interaktionsverläufe gibt.

überblick zur Verteilung der Interaktionsverläufe Tabelle 2 gibt einen Überblick über die Kategorien in Bezug auf die Kitas, in Bezug auf das Geschlecht der Kinderbetreuenden und in Bezug auf das Geschlecht der beteiligten Kinder.

Die Ergebnisse zeigen, dass diejenigen Interaktionsverläufe häufiger sind, bei denen die Kinderbetreuenden das Verhalten der Kinder verstärken, sei es in der Verstärkung des geschlechteruntypischen Verhaltens (Kind undoing gender, Betreuende Dramatisierung mit undoing gender, 40 Sequenzen) oder in der Verstärkung des geschlechterstereotypischen Verhaltens (Kind doing gender, Betreuende Dramatisierung mit doing gender, 34 Sequenzen). Wenn die Interaktionsverläufe nach dem Geschlecht des beteiligten Kindes analysiert werden, zeigen sich etwas mehr undoing gender bei den Mädchen, bei den Jungen etwas mehr doing gender. In Bezug auf das Geschlecht der Kinderbetreuenden zeigt sich, dass Männer einen etwas höheren Anteil für den Interaktionsverlauf undoing gender, Dramatisierung, undoing gender. Es ist jedoch nicht das Ziel des Forschungsprojektes, Unterschiede im Verhalten von Jungen und Mädchen zu erklären oder Unterschiede im Verhalten von Männern und Frauen im Beruf der Kinderbetreuung aufzudecken, das Design wäre dafür nicht geeignet und die Datenbasis zu gering. 
Tabelle 2: Doing, undoing, Dethematisierung und Dramatisierung in Bezug auf die Organisation, das Geschlecht der Betreuenden und das Geschlecht der Kinder

\begin{tabular}{|l|c|c|c|c|c|c|}
\hline & $\begin{array}{c}\text { Kind doing } \\
\text { gender, } \\
\text { Betreuende } \\
\text { Dethe- } \\
\text { matisierung }\end{array}$ & $\begin{array}{c}\text { Kind doing } \\
\text { gender, } \\
\text { Betreuende } \\
\text { Dramati- } \\
\text { sierung } \\
\text { mit doing } \\
\text { gender }\end{array}$ & $\begin{array}{c}\text { Kind doing } \\
\text { gender, } \\
\text { Betreuende } \\
\text { Dramati- } \\
\text { sierung mit } \\
\text { undoing } \\
\text { gender }\end{array}$ & $\begin{array}{c}\text { Kind } \\
\text { undoing } \\
\text { gender, } \\
\text { Betreuende } \\
\text { Dramati- } \\
\text { sierung } \\
\text { mit doing } \\
\text { gender }\end{array}$ & $\begin{array}{c}\text { Kind } \\
\text { undoing } \\
\text { gender, } \\
\text { Betreuende } \\
\text { Dramati- } \\
\text { sierung mit } \\
\text { undoing } \\
\text { gender }\end{array}$ & $\begin{array}{c}\text { Kind } \\
\text { undoing } \\
\text { gender, } \\
\text { Betreuende } \\
\text { Dethemati- } \\
\text { sierung }\end{array}$ \\
\hline $\begin{array}{l}\text { Total pro } \\
\text { Kategorie }\end{array}$ & 6 & 14 & 14 & 40 & 11 \\
\hline Kita & 34 & & & \\
\hline Kita gelb & $1(3 \%)$ & $10(30 \%)$ & $5(15 \%)$ & $4(12 \%)$ & $10(30 \%)$ & $4(12 \%)$ \\
\hline Kita rot & $2(8 \%)$ & $1(4 \%)$ & $3(13 \%)$ & $4(17 \%)$ & $8(33 \%)$ & $6(25 \%)$ \\
\hline Kita grün & $2(9 \%)$ & $9(39 \%)$ & $2(9 \%)$ & $2(9 \%)$ & $7(30 \%)$ & $1(4 \%)$ \\
\hline Kita blau & $1(3 \%)$ & $14(37 \%)$ & $4(11 \%)$ & $4(11 \%)$ & $15(39 \%)$ & $0(0 \%)$ \\
\hline Kinder-betreuende & $3(4 \%)$ & $22(29 \%)$ & $9(12 \%)$ & $6(8 \%)$ & $30(40 \%)$ & $5(7 \%)$ \\
\hline Männlich & $3(7 \%)$ & $12(27 \%)$ & $5(11 \%)$ & $8(18 \%)$ & $10(23 \%)$ & $6(14 \%)$ \\
\hline Weiblich & $3(5 \%)$ & $23(37 \%)$ & $10(16 \%)$ & $4(6 \%)$ & $18(29 \%)$ & $5(8 \%)$ \\
\hline Kind & $3(5 \%)$ & $11(20 \%)$ & $4(7 \%)$ & $10(18 \%)$ & $22(40 \%)$ & $6(11 \%)$ \\
\hline Junge & &
\end{tabular}

Die Verteilung in Tabelle 2 zeigt zudem, dass es Unterschiede zwischen den untersuchten Kitas gibt. Die Abbildung 2 visualisiert die unterschiedlichen Muster.

Während Kita gelb, grün und blau alle einen ähnlichen Verlauf zeigen, mit den beiden Spitzen bei der Verstärkung von doing gender wie auch von undoing gender, zeigt Kita rot einen anderen Verlauf. Bei Kita rot ist die Verstärkung von doing gender sehr wenig ausgeprägt, hingegen die Verstärkung von undoing gender viel mehr als in den andern Kitas. Diese unterschiedlichen Muster wie auch unsere Beobachtungen legen nahe, dass es in den Interaktionen im pädagogischen Alltag der Kinderkrippen Unterschiede in der Organisationskultur gibt.

\section{Diskussion}

Für diesen Beitrag wurde auf die Videodaten fokussiert. Das gesamte Videomaterial wurde gesichtet und mit den ethnographischen Notizen verglichen. Für die detaillierte Analyse wurden hier im Bereich des freien Spiels und der freien Aktivität ausgewählt. Diese Schwerpunktsetzung reflektiert die Betonung der Kinderbetreuenden wie auch der Kita-Leitungen in den Interviews: das freie Spiel wird als zentrales Qualitätsmerkmal von allen hervorgehoben. Auch in der Literatur zur pädagogischen Qualität kommt dem Spiel und der Begleitung des 


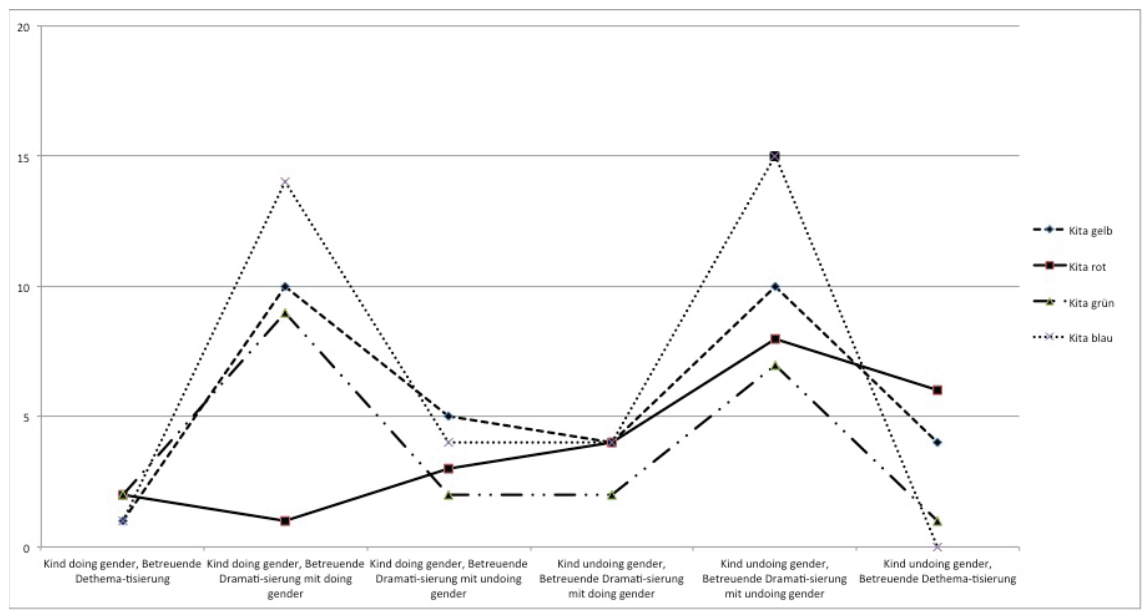

Abbildung 2: Muster der Interaktionsverläufe in Bezug zur Kinderkrippe

Spiels der Kinder durch die Betreuenden grosse Bedeutung zu (Wood, 2011). Geschlecht wird im Alltag der Kinderkrippe auch in andern Praktiken relevant gemacht (Nentwich et al., im Druck), beispielsweise in der Gestaltung der Pflege oder der Mahlzeiten. Die hier analysierten Interaktionsverläufe beginnen mit einer Aktivität oder Aussage des Kindes und umfassen die darauf folgende Reaktion der Kinderbetreuenden. Der weitere Verlauf wird nicht mehr analysiert, es kann davon ausgegangen werden, dass die Kinder die Reaktion wiederum interpretieren, ihr widersprechen oder sie als Ermutigung erleben. Auch gibt das hier gewählte Vorgehen keine Einblicke in die Überlegungen und Überzeugungen der Kinderbetreuenden für die gezeigte Reaktion (Sandström, Stier \& Sandberg, 2013). Die Analyse der Interaktionsverläufe ermöglicht hingegen, doing und undoing gender wie auch Dramatisierung und Dethematisierung in den Alltagspraktiken der Kita zu fassen und damit die Fluidität (Nentwich \& Kelan, 2014) und das ständige Wechseln des relevant Werdens von Gender in der Kinderkrippe zu berücksichtigen.

Die Unterscheidung zwischen dem Verhalten der Kinder und der Reaktion der Kinderbetreuenden im Interaktionsverlauf gibt beispielhaft Einblick, wie Kinderbetreuerinnen und Kinderbetreuer mit ihren situativen Reaktionen auf die Nutzung der kulturellen Ressource Geschlecht durch die Kinder diesen wiederum verschiedene Spielarten von Geschlecht vermitteln. Doing und undoing gender wird subtil im Alltag verstärkt, umgedeutet oder aber nicht beachtet. Es kann vermutet werden, dass die Kinder auch auf diese Art und Weise die Normativität der Geschlechterordnung erfahren, ohne dass diese mit Sanktionen durchgesetzt werden würde (Maihofer, 2002). Welche Wirkungen von doing und undoing gender sowie Dramatisierungen und Dethematisierungen im pädagogischen Handeln der Kinderbetreuenden für die Kinder ausgehen, 
kann in dieser Studie nicht empirisch analysiert werden. Überlegungen darüber, welche Wirkungen dieses Verhalten hat, stützen sich auf theoretische Grundlagen. Im Sinne der Förderung der Gleichstellung werten wir es als positiv, dass die Kinder nicht explizit für geschlechteruntypisches Verhalten mit Hinweisen wie ,du bist ein Junge du solltest $\mathrm{x}$ tun ', ein Mädchen tut y nicht' kritisiert werden. Auch reagieren die Kinderbetreuenden auf undoing gender häufig bestärkend mit Dramatisierung und undoing gender und weniger häufig durch Dramatisierung und doing gender. Dies könnte als Beitrag zur Förderung der Gleichstellung der Geschlechter gewertet werden, da Geschlechterbinaritäten so nicht verstärkt werden.

Insgesamt zeichnet sich bei drei der Krippen das Muster der Interaktionen jedoch dadurch aus, dass das, was die Kinder einbringen, sei es doing oder undoing gender, aufgenommen und verstärkt wird. Weniger häufig wird dem doing gender eines Kindes ein undoing gender in der Reaktion der Kinderbetreuenden entgegengesetzt. Dies steht möglicherweise in Zusammenhang mit den pädagogischen Überzeugungen der Kinderbetreuenden. Die Betonung des freien Spiels kann auch dazu führen, dass eine aktive Beteiligung der Kinderbetreuenden als negativ angesehen wird und Gesprächsanlässe, die das Relevant Machen von Gleichstellung im Alltag bieten würden, nicht genutzt werden. Dass sich die Kinderbetreuenden stark daran orientieren, was die Kinder einbringen und dies verstärken, sei es nun den geschlechtsstereotypen Erwartungen entsprechend oder aber widersprechend, lässt die Vermutung zu, dass es für eine geschlechterreflektierende Praxis alltagsnahe Trainings und Reflexion bräuchte, die zudem auf ihre Wirkung hin untersucht werden müssten. Wissenschaftliche Interventionsstudien zur Wirkung einer geschlechterreflektierenden Praxis in der frühen Kindheit fehlen jedoch bisher gänzlich.

Die Muster der Interaktionsverläufe unterscheiden sich zwischen den Kitas. Während in drei Kitas das von den Kindern Eingebrachte verstärkt wird, geschieht in Kita rot verhältnismässig wenig Verstärkung von doing gender, jedoch mehr Verstärkung von undoing gender. Auch zeigen die Kinder in Kita rot insgesamt weniger doing gender, sie engagieren sich weniger in geschlechterstereotypen Aktivitäten. Möglicherweise liegt hier eine Wechselwirkung vor: Die Kinderbetreuenden verstärken undoing gender vermehrt, doing gender weniger. Die Kinder fühlen sich stärker ermutigt, nicht geschlechterstereotyp zu spielen. Auch in anderen Bereichen unserer Analyse zeigt sich, dass Kita rot undoing gender ermöglicht und doing gender abschwächt. In der Analyse der Räume fiel auf, dass sich Kita rot in der Raumgestaltung von andern Kitas unterscheidet: Kita rot hat keine räumlich getrennten Bauecken und Puppenstuben eingerichtet, die Verkleidungen bieten weiblich und männlich konnotierte Rollen an, und die Kita legt Wert auf unstrukturiertes Material und Materialien zum Forschen und Entdecken. Es ist darum denkbar, dass diese geschlechterreflektierende Gestaltung der Räume dazu führt, dass die Kinder insgesamt weniger den Geschlechterstereotypen gemäss ihre Spielaktivitäten wählen. In Umsetzungs- 
projekten in der Folge dieses Forschungsprojektes werden darum besonders auch die räumliche Gestaltung und das Spielangebot reflektiert (Vogt, Nentwich \& Tennhoff, im Druck). Dass unterschiedliche Muster der Interaktionsverläufe zwischen Kita rot und den andern Kitas möglich sind, deutet auf die Bedeutung von Organisationskultur für die geschlechterreflektierende Praxis in Kitas hin. Für Gleichstellung in der Kita sind darum die Leitungspersonen und die Teams im Sinne einer Organisationsentwicklung gefragt.

Die detaillierte Analyse der Interaktionsverläufe zwischen Kindern und Kinderbetreuenden zeigt auf, wie Geschlecht als kulturelle Ressource in den pädagogischen Alltagsinteraktionen in der Kita relevant gemacht wird. Die Kinder zeigen beides, doing und undoing gender, die Kinderbetreuenden reagieren darauf mit Dramatisierung oder Dethematisierung. Insgesamt verstärken sie häufig das, was die Kinder zeigen. Für eine geschlechterreflektierende Praxis in den Kitas und damit für einen Förderung der Gleichstellung wäre jedoch eine weitergehende Klärung notwendig, was pädagogische Qualität in der Begleitung von freiem Spiel und freien Aktivitäten ausmacht. Die Interaktionsverläufe können zur Reflexion der pädagogischen Praxis genutzt werden.

\section{Anmerkungen}

1 Das Forschungsprojekt wurde im Rahmen des NFP60 vom Schweizerischen Nationalfonds unterstützt (Projektnummer 406040_129314). Wir danken den beteiligten Kitas für die Teilnahme.

\section{Literatur}

Ärlemalm-Hagsér, E. (2010) Gender choreography and micro-structures - early childhood professionals' understanding of gender roles and gender patterns in outdoor play and learning. European Early Childhood Education Research Journal, 18(4), 515-525.

Bilden, H. (1991). Geschlechtsspezifische Sozialisation. In K. Hurrelmann \& D. Ulich (Hrsg.), Neues Handbuch der Sozialisationsforschung (4. Auflage, S. 279-301). Weinheim: Beltz.

Börding, M. (2009). Eigenen Stereotypen auf der Spur. Gender-Trainings für Lehrerinnen und Lehrer - eine Annäherung. Grundschule, 41(9), 32-34.

Brandes, H., Andrä, M., Röseler, W. \& Schneider-Andrich, P. (2013). Männer in Kitas Was machen sie anders und wie profitieren die Kinder von ihnen? Ergebnisse aus der "Tandem-Studie» zu professionellem Erziehungsverhalten von Männern und Frauen. Frühe Kindheit, 16(5), 38-43.

Breidenstein, G. \& Kelle, H. (2003). Mädchen und Jungen und Kinder-Kontexte der Geschlechterunterscheidung in der Schule. Weinheim: Beltz.

Browne, N. (2004). Gender equity in the early years. Maidenhead: Open University Press.

Butler, J. (2004). Undoing Gender. Routledge: London.

Chick, K. A., Heilman-Houser, R. A. \& Hunter, M. W. (2002). The impact of child care on gender role development and gender stereotypes. Early Childhood Education Journal, 29(3), 149-154.

Deutsch, F. (2007). Undoing Gender. Gender \& Society, 21(1), 106-127.

Faulstich-Wieland, H. (2001). Gender Mainstreaming im Bereich der Kindertagesstätten. In G. von Ginsheim \& D. Meyer (Hrsg.), Gender Mainstreaming. Neue Perspektiven für die Jugendhilfe (S. 121-132). Berlin: Stiftung SPI. 
Faulstich-Wieland, H., Weber, M. \& Willems, K. (2004). Doing gender im heutigen Schulalltag. Empirische Studien zur sozialen Konstruktion von Geschlecht in schulischen Interaktionen. Weinheim: Juventa.

Freeman, N. K. (2007). Preschoolers' perceptions of gender appropriate toys and their parents' beliefs about genderized behaviors: Miscommunication, mixed messages, or hidden truths? Early Childhood Education Journal, 34(5), 357-366

Gherardi, S. (1995). Gender, symbolism and organizational cultures. London: Sage.

Giraldo, E. \& Colyar, J. (2012). Dealing with gender in the classroom: a portrayed case study of four teachers. International Journal of Inclusive Education, 16(1), 25-38.

Grünewald-Huber, E. \& von Gunten, A. (2009). Werkmappe Genderkompetenz: Materialien für geschlechtergerechtes Unterrichten. Zürich: Verlag Pestalozzianum.

Goffman, E. (1994). Das Arrangement der Geschlechter. In H. A. Knoblauch (Hrsg.), Interaktion und Geschlecht (S. 105-158). Frankfurt/Main, New York: Campus Verlag. Henschel, A. (2014). Geschlechtersozialisation in der frühen Kindheit In R. Braches-Chyrek, C. Röhner, H. Sünker \& M. Hopf (Hrsg.), Handbuch Frühe Kindheit (S. 97-107). Opladen: Budrich.

Hyun, E. \& Choi, D. H. (2004). Examination of young children's gender-doing and genderbending in their play dynamics: A cross-cultural exploration. International Journal of Early Childhood, 36(1), 48-64.

Hirschauer, S. (2001). Das Vergessen des Geschlechts. Zur Praxeologie einer Kategorie sozialer Ordnung. In B. Heintz (Hrsg.), Geschlechtersoziologie (S. 209-235). Wiesbaden: Westdeutscher Verlag.

Hollander, J. A. (2013). «I Demand More of People». Accountability, Interaction, and Gender Change. Gender \& Society, 27(1), 5-29.

Kelan, E. (2010). Gender Logic and (Un)doing Gender at Work. Gender, Work and Organization, 17(2), 174-194.

Kommission für Wissenschaft, Bildung und Kultur des Nationalrates (12.08.2014) Parlamentarische Initiative Weiterführung und Weiterentwicklung der Finanzhilfen für familienergänzende Kinderbetreuung Bericht 13.451 Zugriff am 31.01.2015 unter http://www. admin.ch/opc/de/federal-gazette/2014/6619.pdf

Krabel, J. \& Cremers, M. (2008). Gender Loops - Praxisbuch für eine geschlechterbewusste und -gerechte Kindertageseinrichtung. Berlin: DissenseV. Zugriff am 31.01.2015 unter http:// www.genderloops.eu/de/

Kuger, S., Kluczniok, K., Sechtig, J. \& Smidt, W. (2011). Gender im Kindergarten. Empirische Datenlage zu Unterschieden zwischen Mädchen und Jungen. Zeitschrift für Pädagogik, 57(2), 269-288.

MacNaughton, G. (2006). Constructing gender in early-years education. In C. Skelton, B. Francis \& L. Smulyan (Eds.), The SAGE Handbook of Gender and Education (pp. 127-138). London: Sage.

Maihofer, A. (2002). Geschlecht und Sozialisation. Eine Problemskizze. Erwägen, Wissen, Ethik, EWE (vormals EuS), 13(1), 13-26.

NFP60 Gleichstellung der Geschlechter (2014). Ergebnisse und Impulse. Synthesebericht. Bern: Schweizerischer Nationalfonds. Zugriff am 31.01.2015 unter http://www.nfp60. ch/SiteCollectionDocuments/NFP_60_Synthese_de_Web.pdf

Nentwich, J. C. \& Kelan, E. K. (2014). Towards a topology of 'doing gender': An analysis of empirical research and its challenges. Gender, Work \& Organization, 21(2), 121-134.

Nentwich, J. C., Vogt, F. \& Tennhoff, W. (im Druck). Care and education? Exploring the gendered rhythms and routines of childcare work. In B. Liebig, K. Gottschall \& B. Sauer: Gleichstellung der Geschlechter, NFP 60.

Nentwich, J. C. \& Vogt, F. (2014). Puppenstuben, Bauecken, Waldtage: (Un)doing Gender in der Kinderkrippe. Zusammenfassung der Projektergebnisse - Langversion. Bern: Schweizerischer Nationalfonds. Zugriff am 31.1.2015 unter http:/www.nfp60.ch/SiteCollection Documents/Projekte/nfp60_projekte_nentwich_zusammenfassung_projektergebnisse_lang.pdf 
OECD (2012). Education at a Glance. OECD Indicators. Paris: OECD Publishing.

Ostrov, J. M. \& Hart, E. J. (2013). Observational methods. In T. D. Little (Ed.), The Oxford Handbook of Quantitative Methods (Vol. 1: Foundations, pp. 285-303). Oxford: Oxford University Press.

Papadopoulou, M. (2012). The ecology of role play: intentionality and cultural evolution. British Educational Research-Journal, 38(4), 575-592.

Paseka, A. (2008). Wie Kinder zu Mädchen und Buben werden. Einige Erkenntnisse aus der Sozialisations- und Geschlechterforschung. In M. Buchmayr (Hrsg.), Geschlecht lernen. Gendersensible Didaktik und Pädaogigik (S. 15-31). Innsbruck: Studienverlag.

Pickering, M. (2001). Stereotyping: The Politics of Representation. New York: Palgrave.

Quali-Kita (2014). Quali-Kita-Handbuch. Standard des Qualitätslabels für Kindertagesstätten. kibesuisse, Verband Kinderbetreuung Schweiz (Hrsg.). Zugriff am 31.01.2015 unter http://quali-kita.ch/fileadmin/user_upload/Arbeitsinstrumente/QualiKita_Handbuch.pdf

Rabe-Kleberg, U. (2003). Gender Mainstreaming und Kindergarten. Weinheim: Beltz.

Rendtorff, B. (2006). Geschlechtstypisierende Aspekte im Kinderleben. In S. Andresen \& I. Diehm (Hrsg.), Kinder, Kindheiten, Konstruktionen (S. 135-145). Wiesbaden: VS Verlag für Sozialwissenschaften.

Rendtorff, B. (2011). Was Kinder spielen, wenn sie spielen - geschlechtstypische Aspekte im kindlichen Spiel. In M. Klaas, A. Flügel, R. Hoffmann \& B. Bernasconi (Hrsg.), Kinderkultur(en) (S. 71-83). Wiesbaden: VS Verlag für Sozialwissenschaften.

Sandström, M., Stier, J. \& Sandberg, A. (2013). Working with gender pedagogics at 14 Swedish preschools. Journal of Early Childhood Research, 11(2) 123-132.

SKBF (2014). Bildungsbericht Schweiz 2014. Aarau: Schweizerische Koordinationsstelle für Bildungsforschung.

Stern, S., Iten, R., Schwab, S., Felfe, C., Lechner, M., Thiemann, P. (2013) Familienergänzende Kinderbetreuung und Gleichstellung Schlussbericht. Zürich/ St. Gallen. Zugriff am 31.01.2015 unter http://www.nfp60.ch/SiteCollectionDocuments/Projekte/nfp60_iten_ schlussbericht_d.pdf

Vogt, F. (im Druck). Bildung in der frühen Kindheit: ,Bildung' und ,pädagogische Qualität' als Auslöser von Angebotsausbau und Innovation. In M. Bergman, H. U. Grunder, S. HupkaBrunner \& C. Imdorf (Hrsg.), Qualität in der Bildung. Bad Heilbrunn: Klinkhardt Verlag.

Vogt, F., Nentwich, J. \& Tennhoff, W. (im Druck). Puppenecken und Bauecken: Gender und Raumordnung. In C. Müller, L. Amberg, T. Dütsch, E. Hildebrand, F. Vogt \& E. Wannack (Hrsg.), Perspektiven und Potenziale der Schuleingangsstufe. Münster: Waxmann.

West, C. \& Zimmerman, D. (1987). Doing Gender. Gender and Society, 1(2), 125-151.

West, C. \& Zimmerman, D. H. (2009). Accounting for doing gender. Gender and society, 23(1), 112-122.

Wetterer, A. (2008). Geschlechterwissen \& soziale Praxis. Grundzüge einer wissenssoziologischen Typologie des Geschlechterwissens.In A. Wetterer (Hrsg.) Geschlechterwissen und soziale Praxis: Theoretische Zugänge - empirische Erträge, (S. 39-63). Sulzbach: Taunus.

Wood, E. (2011). Entwicklung einer integrierten Pädagogik für die frühe Bildung. In F. Vogt, M. Leuchter, A. Tettenborn, U. Hottinger, M. Jäger \& E. Wannack (Hrsg.). Entwicklung und Lernen junger Kinder (S. 123-132). Münster: Waxmann Verlag.

Wustmann Seiler, C. \& Simoni, H. (2012). Orientierungsrahmen für frühkindliche Bildung, Betreuung und Erziehung in der Schweiz. Erarbeitet vom Marie Meierhofer Institut für das Kind, erstellt im Auftrag der Schweizerischen UNESCO-Kommission und des Netzwerks Kinderbetreuung Schweiz. Zürich. Zugriff am 31.01.2015 Unter http://www.orientierungsrahmen.ch

Schlagworte: Gender, frühkindliche Bildung, Kinderbetreuung, Alltagspraxis, Videostudie 


\section{Doing et undoing gender dans les crèches: une analyse des interactions des éducateurs/éducatrices avec les enfants}

\section{Résumé}

Pour nombreux enfants, la crèche est la première institution éducative qu'ils fréquentent. La crèche donc est importante par rapport à la question de l'éducation à l'égalité des sexes. Dans cet article, sur la base d'une étude ethnographique et de données vidéo effectuées dans quatre crèches, nous examinons comment les questions de genre s'actualisent dans les pratiques pédagogiques quotidiennes. Le codage des interactions a été réalisé à partir des concepts de doing gender, undoing gender, dramatisation et dé-thématisation. Les résultats montrent que les éducateurs et éducatrices renforcent les comportements des enfants et exigent rarement l'égalité. Pour soutenir la question de l'éducation à l'égalité dans les crèches, la culture d'organisation et la qualité de l'éducation s'avèrent être primordiales.

Mots-clés: Genre, éducation de la petite enfance, pratiques pédagogiques quotidiennes, éducateurs de la petite enfance, analyse de vidéo

\section{Doing e undoing gender nella scuola dell'infanzia. Analisi di dati video dell'interazione tra educatori e bambini.}

\section{Riassunto}

Per molti bambini, la scuola dell'infanzia è la prima istituzione scolastica, importante dunque per la parità dei sessi. Sulla base di uno studio etnografico basato su video nelle scuole dell'infanzia della Svizzera tedesca, questo contributo si interroga su come il genere acquisti rilevanza nella pratica pedagogica quotidiana degli educatori e delle educatrici. Per codificare i dati video le interazioni sono state analizzate secondo concetti doing gender, undoing gender, drammatizzazione e de-tematizzazione. I risultati mostrano che gli educatori e le educatrici rinforzano il comportamento mostrato dai bambini, ma raramente esigono eguaglianza. Per sostenere l'uguaglianza nella scuola dell'infanzia sono fondamentali la cultura dell'organizzazione e la qualità dell'educazione.

Parole chiave: Genere, educazione della prima infanzia, pratiche pedagogiche quotidiane, educatori della prima infanzia, analisi di video 


\title{
Doing and Undoing Gender in the nursery: a video analysis of the interactions of early childhood educators with children
}

\begin{abstract}
Summary
Many children experience nurseries as the first institutional, educational setting, therefore they are relevant for ensuring gender equality. Four Swiss nurseries were visited for an ethnographic, video-based study in order to examine how gender is made relevant in the day-to-day practices of early childhood educators. The video data was analysed looking at the sequence of interaction between child and educator in terms of doing and undoing gender, as well as dramatizing and de-thematising. The results of the video study indicate that early childhood educators enhance the behaviour of the children, be it doing or undoing gender. Reactions seeking to enhance gender equality and challenging doing gender are however rare. In order to foster gender equality in nurseries, the organisational culture and quality are paramount.
\end{abstract}

Keywords: Gender, early childhood education and care, daily practices, video analysis, gender stereotypes 
